University for Business and Technology in Kosovo

UBT Knowledge Center

UBT International Conference

2015 UBT International Conference

Nov 7th, 9:00 AM - 5:00 PM

\title{
Processing of GIS data and building of 3D dem models with FME transformer tools
}

\author{
Hebib Alili \\ University for Business and Technology, hebib.alili@ubt-uni.net \\ Fadil Shehu \\ University for Business and Technology, fadilshehu01@hotmail.com \\ Shenur Muslija \\ University for Business and Technology, shenur@gmail.com
}

Follow this and additional works at: https://knowledgecenter.ubt-uni.net/conference

Part of the Civil Engineering Commons, and the Structural Engineering Commons

\section{Recommended Citation}

Alili, Hebib; Shehu, Fadil; and Muslija, Shenur, "Processing of GIS data and building of 3D dem models with FME transformer tools" (2015). UBT International Conference. 78.

https://knowledgecenter.ubt-uni.net/conference/2015/all-events/78

This Event is brought to you for free and open access by the Publication and Journals at UBT Knowledge Center. It has been accepted for inclusion in UBT International Conference by an authorized administrator of UBT Knowledge Center. For more information, please contact knowledge.center@ubt-uni.net. 


\title{
Processing of GIS data and building of 3D dem models with FME transformer tools
}

\author{
Hebib Alili ${ }^{1}$, Fadil Shehu ${ }^{2}$, Shenur Muslija ${ }^{3}$ \\ UBT - High Education Institution \\ hebib.alili@ubt-uni.net ${ }^{1}$, fadilshehu01@hotmail.com², shenur@gmail.com ${ }^{3}$
}

\begin{abstract}
The processes of processing of 2D or 3D geodetic or geo-information data by using the FME software and other software's or tools in combination and then through mathematical logics in FME building of such 3D models from original measured terrain data within transformers given by FME, could be considered as very important attempt of creating of such 3D models, exactly when we have to deal with huge data by the size of its as input data. These kind of doing of processing of data through FME software, can be considered as important issue also for resolving of other today related exercises derived from field of geodesy and geo-information. By the way many organizations and private companies or investors require realistic and accurate 3D models of their projects for decisionmaking through visualization and simulation. Additionally for creating the most accurate 3D models as the final product, both $2 \mathrm{D}$ and $3 \mathrm{D}$, often needs to be shared with decision-makers in a different file format.
\end{abstract}

Keywords: 3D DEM, GIS, GPS, DEM, SRTM, RASTER, 3Dpdf.

\section{Introduction}

\subsection{General Information about FME (Feature Manipulation Engine)}

A short history of Safe FME software: Safe Software was founded in 1993, since there was a need to work with a format known as the Spatial Archive and Interchange Format (SAIF) which was very similar to GML. Since "SAIF" was considered "safe," a decision was made to name the software "Safe Software" as the main reason to work with the SAIF format files. In 1993, when the company was founded, the focus was on the translation technology from/into the SAIF format. As they went forward, they realised that other formats could benefit from this technology. By 1996, they had released the first version of their data translation software: the Feature Manipulation Engine. By 2008, they had shortened it to "FME" and since then FME software advanced for support and works on manipulation for different kinds of file formats. FME today has a huge gallery of different transformation functions, known as tool transformers for translation from/into different file formats. Currently, FME supports data in over 300 formats across spatial and non-spatial data ty pes including GIS, CAD, raster, database, 3D, XML, point cloud, and other. 


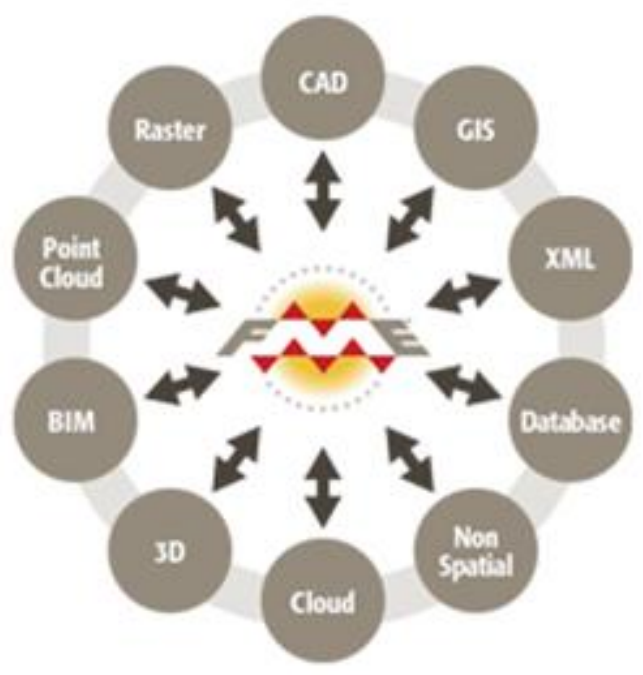

Fig. 1. Safe FME: Exchange formats

The main solutions provided for the transformation of data and formats:

- Applications: CAD products, Bentley products, Erdas Imagine, Intergraph products, ESRI, Google Earth maps, and more.

- Databases: Microsoft SQL Server, Oracle Spatial, PostGIS, Teradata, Google Fusion Tables and Netezza Spatial

- Data types: 3D, CAD and GIS Data Exchange, KML, LiDAR- Point Clouds, Metadata, Non-Spatial, PDF, Raster, Sensor Data, XML etc.

\section{Solving the simple exercises by using the FME software.}

\subsection{Solving a simple case: "Processing of collected data with GPS tracking measurements" using FME software for GIS purposes.}

Through these simple exercises we are going to describe the post-processing automatized data processing for our case of data collection with a hand GPS (tracking record method) for GIS implementation projects.

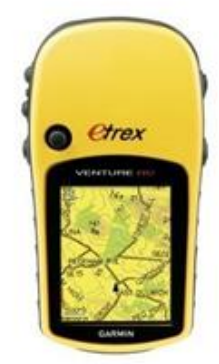

Fig. 2.1. Hand GPS (eTrex)

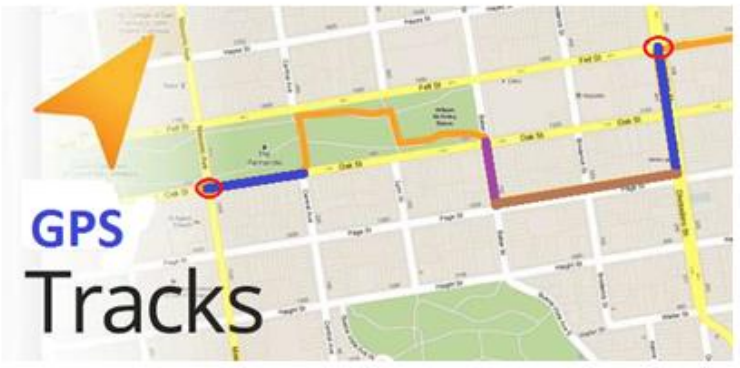

Fig. 2.2. Way point of collected track point with GPS 


\subsection{Technical approach of the case}

The necessary data will be collected in the field (data measurement with a hand GPS using the tracking method) and the recorded tracks or mark point, e.g. measured city roads, places of interest or similar data. These records will be achieved by registering the priority attributes of these data measurements from hand GPS equipment with accuracy $1-10 \mathrm{~m}$ and by applying the tracking method for measurements. Before the starting of measurements in the field the data will need to be well prepared in advance, knowing the possibilities of GPS installed manufacturer software and its possibilities to make records acceptable for registering such data including these attributes or other relevant information. This preparation form, for recording measurements, will be required for the next processing of these measured data in the office. Secondly, prior to the data post-processing, the data need to be prepared in a such a way, that the input data formats has to be well sorted and formatted in order to be an acceptable data format of its software or tools, which will be used in the next process of data post-processing. These data can be post-processed using primitive incorporating methods and by linking them with the collected GPS track data, point by point, using different software such as AutoCAD, GIS tools or other programmable tools. Processing of these data in this form will be wellprepared, but this will not be as fully automated process. By using the FME software and its possibilities, the user will be able to handle, well manage and very quickly conduct the postprocessing of the GPS collected data and also get the required output data format. This output format can then be used as a solution for feature implementation in other projects such GIS implementation or in other types of interpretation for other projects.

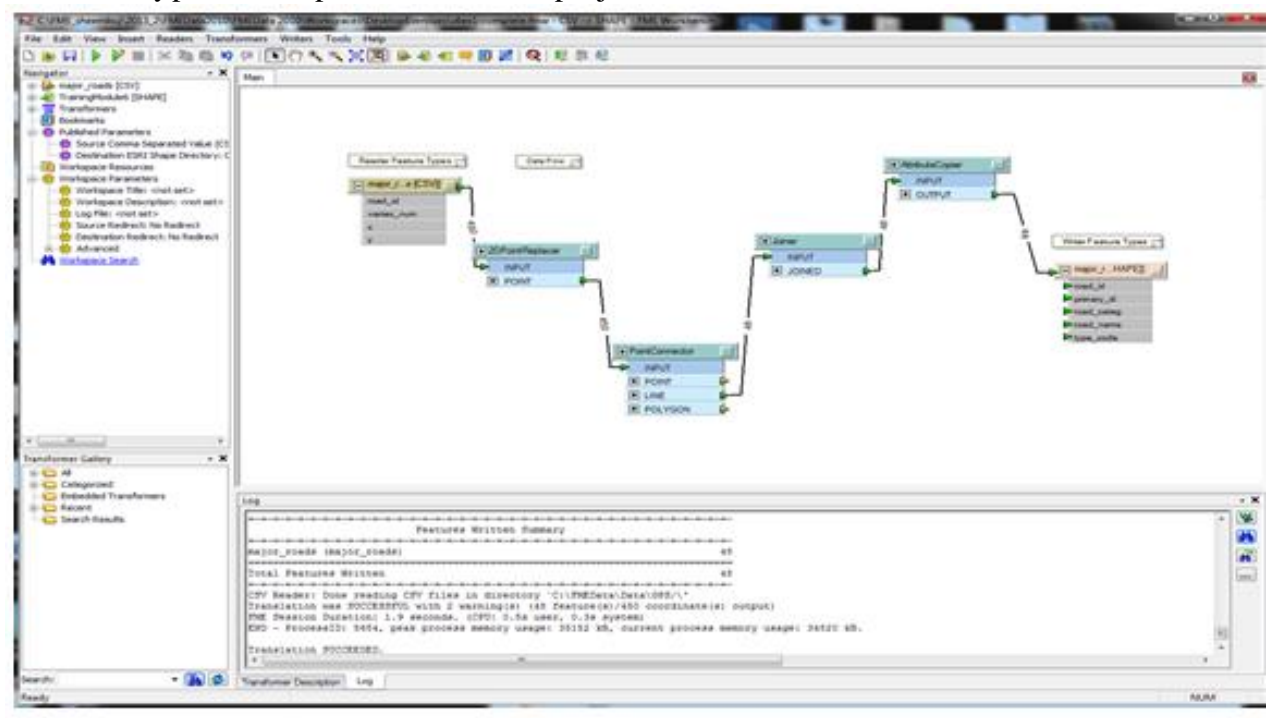

Fig. 3. Workflow: Functional post-processed flows

The FME software is exclusively specialised for reading "Input" of data from different software formats such as GIS, design software, graphical software and other data used nowadays, as well as for writing "Output" for different data file formats. The programming process is related in between input and output in its whole process, where the designer or programmer will make a solution by using of logical operations from the priory predefined FME tools, known as functional transformer tools such as transformers' calculators, raster's, surfaces etc. The project finalisation with a programmable solution using these functional FME predefined transformers tools is known as Functional postprocessed flows. 


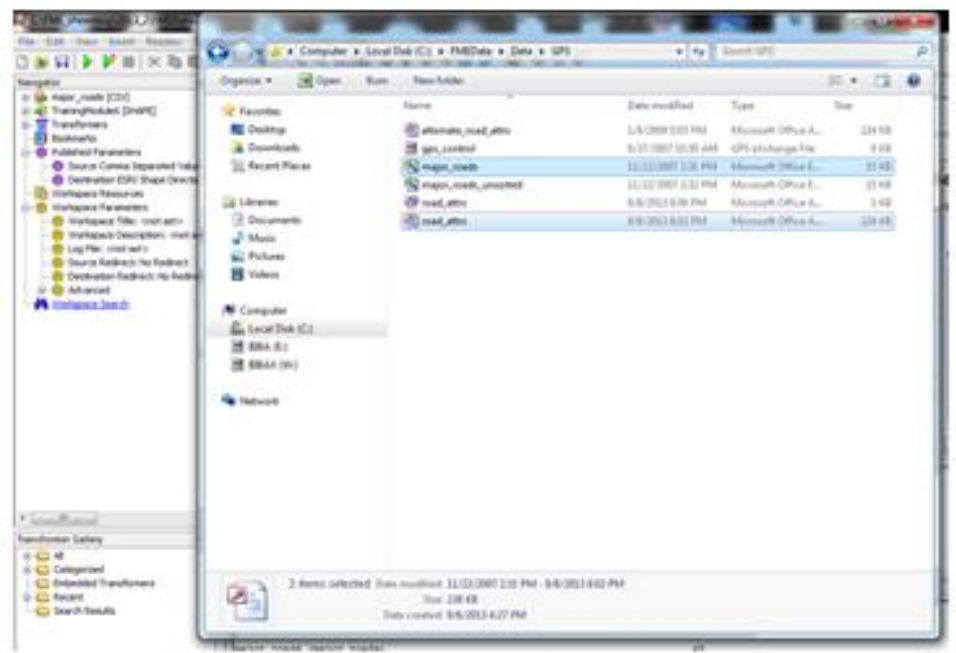

Fig. 3.1. Preparation of data before processing at workflow

Next step, our measured data has to be prepared in advance as a proper file format for the further processing within the FME functional transformers. The whole process of conducting the processing flows will depend on our logical solution, and secondly, should be adoptive with our data to be used in the FME transformers as they by themselves are created. The solution in FME will also depend on the way the workflow process has to be conducted or work without any mistake if we have already performed a good logical solution (through functional FME tools) and this logical solution has to produce our requested preferable output file format. In our case these handled data are prepared in classic software such as text editors or Microsoft products (Excel 2007 or 2010). These GPS data measurements are well sorted and prepared in a form of table and these records by themselves present the information of geodetic coordinates, Id number, attribute of utilities etc. In addition, it will be required that these data are transformed or processed for a prior database file format such as Access table database, known as ".mdb" file format. In this table database, data will be prepared in advance as sorted data information, where then this data will be presented and prepared for feature interpretation as interlink relation due to the work flow process in FME software. Further, the main feature information will present the main information for the ty pe of the road recorded, road ID, road category, attribute of direction, road name etc.

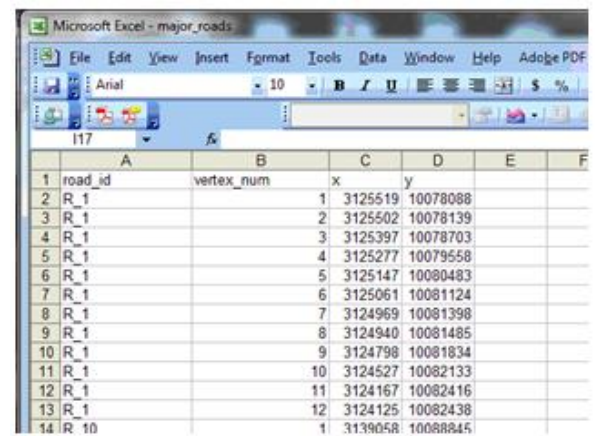

Fig. 4.1. Measured data in excel table

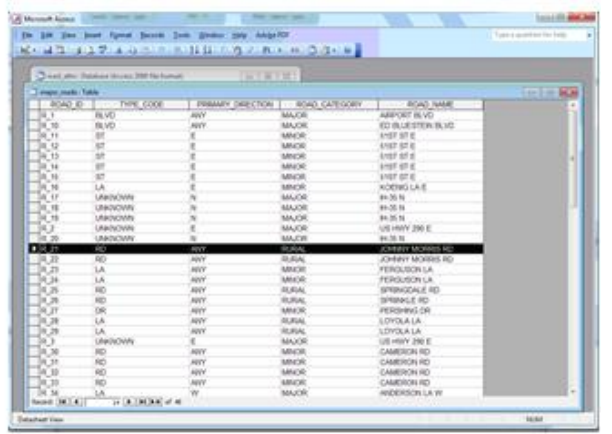

Fig. 4.2. Preparation of data for access table

\subsubsection{The workflow process of creating a solution by FME tools for our case.}

Firstly, in FME we will read our earlier prepared data in excel format within the FME reading tool for ".csv" file formats. The data of input file formats always have to be accordingly formatted for 
FME data flow process and because of this fact, our data from the very beginning of preparation should be prepared for acceptable formats of the calculus in the flows process. For a better explanation, we have to think what data will be used in further calculations, such as numerical data, and what data will be presented as attribute-only, such as non-calculus data as character type of data.

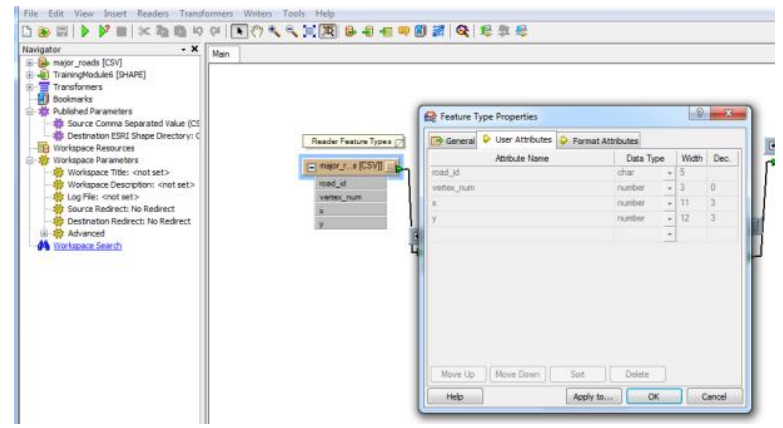

Fig. 5. FME Reader tool for excel data

Having in consideration of these preconditions, for our case as calculus data, will be used 2D coordinates of each GPS measured points represented from origin of ".csv" excel file as coordinates $\mathrm{Xi}$ and $\mathrm{Yi}$ and for the transformer for these process in the workflow, will be used the "2DPointReplacer" FME transformer tool. These coordinates Xi, Yi at the "2DPointReplacer" as input parameters has to be defined as integer number values.

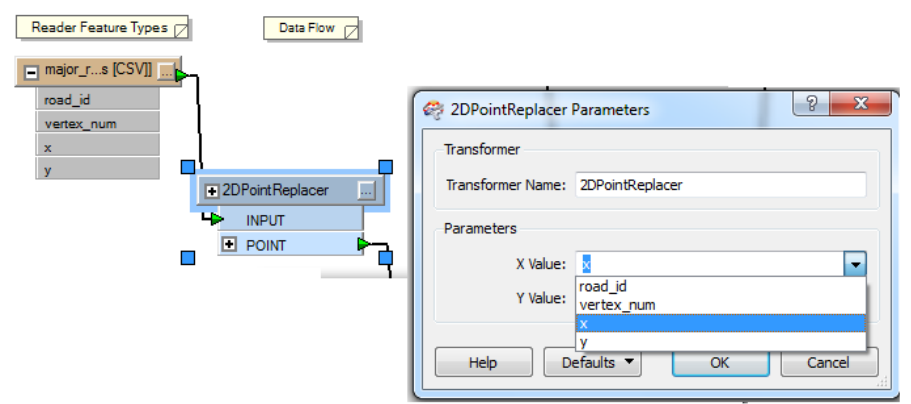

Fig. 6. FME “2DPointReplacer” transformer tool

In the next step forward, for the process of creating of relationship function in between of each priory defined point feature at the origin of ".csv" excel file, will be used the "PointConnector" FME transformer tool. By this, it means that will be created the connections according to the data relation of the measured ID numbers and the relation of its defined attributes, where these attributes are represented as feature at the origin of ".csv" excel file. From these kind, of forming of such relationship, will be possible to be created the new line features (e.g. line measured roads) as output file format. Hence, the input data origin of the "PointConnector", which already comes from the output of "2DPointReplacer", are the 2D coordinates(Xi, Yi) of each tracking GPS points. Based on these, the imaginational GPS measured line collections by its characteristics, will be the feature lines which will derived from the output of the "PointConnector". These feature lines will be related on the information presented for the type of feature data and characteristics information presented for the attribute of feature data. 


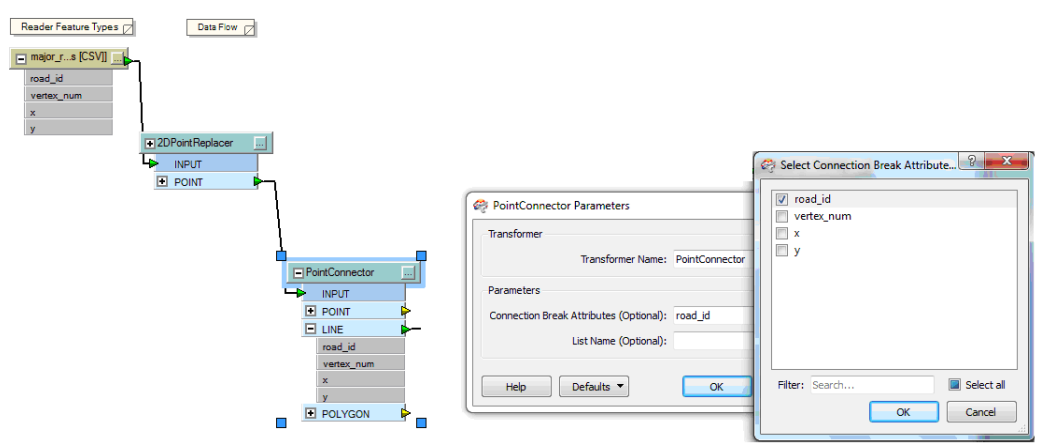

Fig. 7. FME "PointConnector" transformer tool.

After this stage will start the process of joining the attribute definition for each line feature characterised with the data presented in the access database table by using the "Joiner" FME transformer tool.

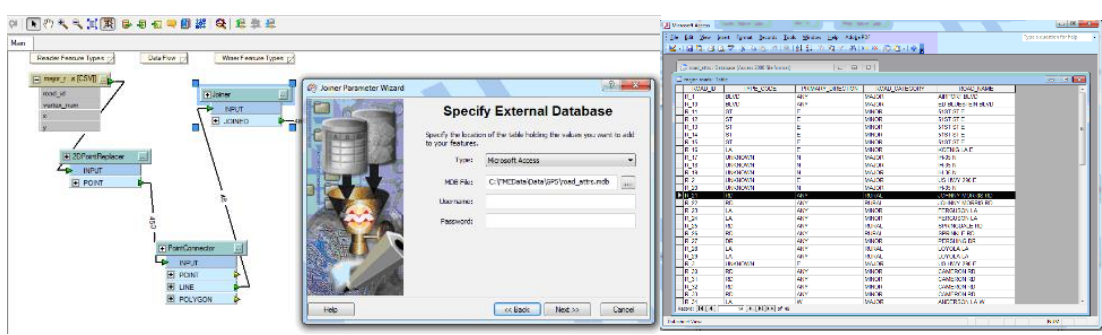

Fig. 8.1. FME "Joiner" transformer tool.

Fig. 8.2. External database(access table).

In this step will be created the connections in between the ".csv" excel table data format and ".mdb" access table data format and also well defined their interrelationship. Firstly, through wizard it is needed to be created a connection with external databases(created database table for our GPS measured data), than the next step, is to be created the data interrelationship in between two different table data formats. By defining the primary keys in both tables, it is easiest of doing the equity or relation in between of these two different tables. These interrelations will be done by defining the matches by equity of key ID numbers in two different data table formats of files.

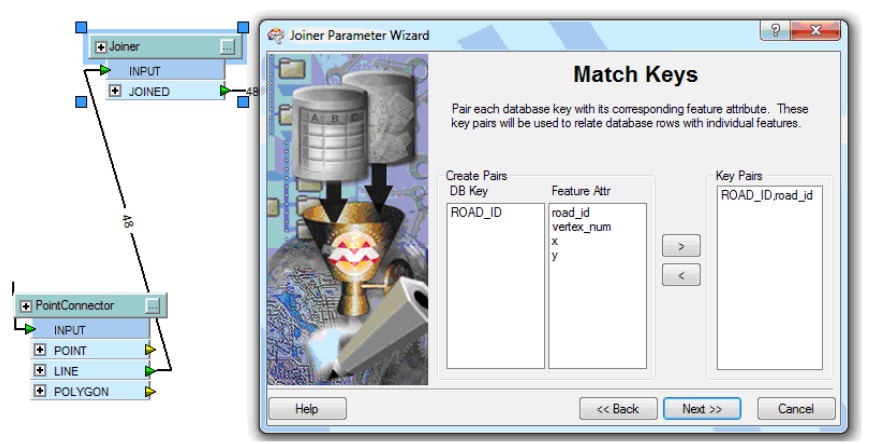

Fig. 9. Matching through equity of keys; FME “Joiner” tool.

Upon completing these matches and the defining process we are ready to copy all data according to the definition on processes of joining the data. These will be done using another transformer tool called "AttributteCopier" which will create new attribute-data from old attribute-data and by copying the rest of these data in order to create the new feature attribute-data. 


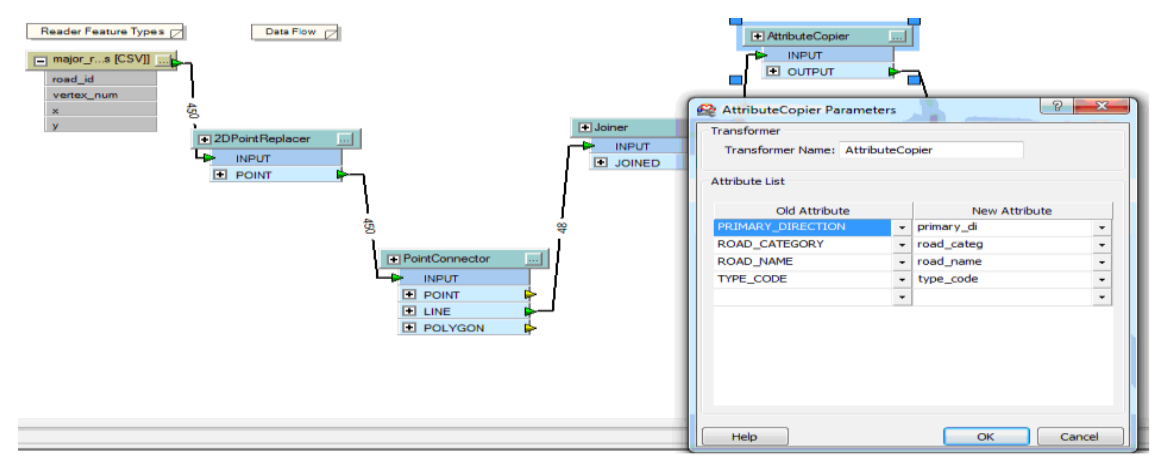

Fig. 10. FME “AttributteCopier” transformer tool.

Before of finalising the solution in workflow, we need to decide, what kind of output source data is needed or requested. Because of this, within the FME, it will be needed to be created the desired or requested output source file and these can be done through the FME "output writers" tools. The desired output data source file, for our case, is preferable the Esri shape file format and the output will be done with the FME "Esri shape writer" tool.

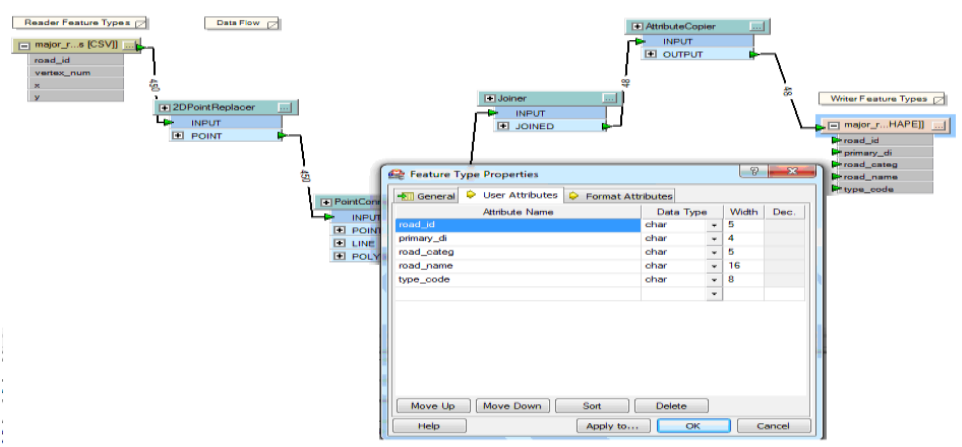

Fig. 10.1. FME "Esri shape writer" tool.

By running of our created solution in the FME and if no error found due to the processing, the data will be created in vector shape file format ( known as Esri shape file). These kind of vector file format we can open it from other dedicated software or editor reader for Esri shape files(.shp file).

Here we see, that conducting a logical operation and using already sophisticated tools such FME transformers, we can fully automatize the process and very quickly produce the required data file format. And this produced file will be ready for use in other work or other implementations such as in GIS implementation or other relevant software to be used for other purposes.

Results: vector shape file(.shp file). 


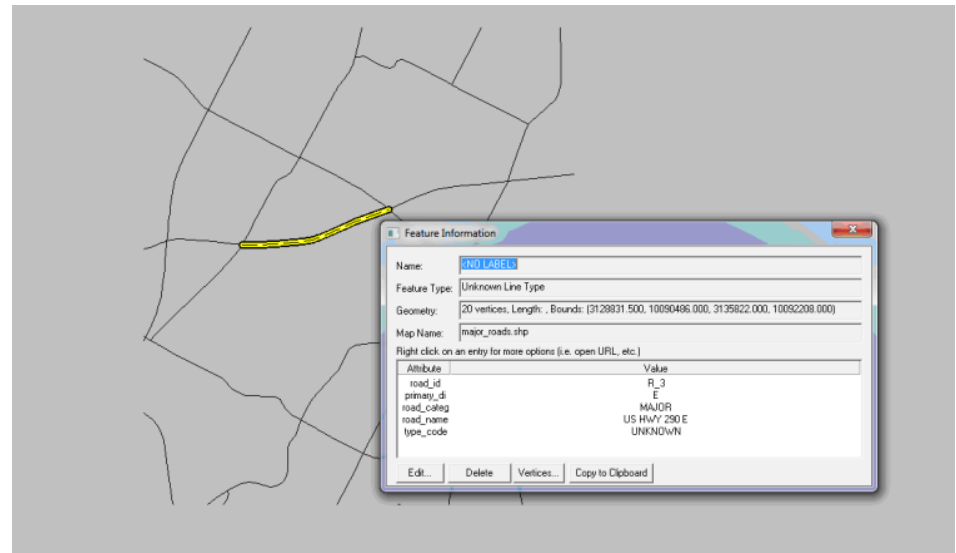

Fig. 11. GM one of software's which can read the shape file formats.

\section{Solving the complex cases within the FME solutions}

\subsection{Creation of DEM(Digital Elevation Model) data surface with overlapping of orthogonal projected Google earth image photo, for a bigger territory by using existing the SRTM(Shuttle Radar Topography Mission) DEM data (3 Arc degrees)}

Preparation of existing data sources SRTM data model: The input data are to be used by DEM data sources which we can get from public data source of ORG. For making a DEM file, the SRTM file format data should be read by relevant software designated for processing this kind of format data and then be converted for output as DEM file format. A useful and sophisticated software for the preparation of this kind of data is "Global Mapper" software (GM). The next goal is to exploit these data in process of automatizing through FME functions tools. For testing purposes, to show that data with large size formats can be used for processing and producing a DEM surface file format, we will use SRTM DEM source file for the territory of Kosovo. For creating DEM surfaces from especially large size of format such as SRTM files for today's software in use, it would be impossible to make such processing and create these surfaces with an overlapped photo image. However, for smaller pieces of these kinds of data (SRTM data files), SRTM data with much smaller sizes than our test territory can be processed without any problems with today software designated for creating DEM surfaces with overlapped photo image. In next explanation we want to show that these kinds of data with greater size formats can be processed without any problem, but only by using the solution given from functional FME tools. Firstly, we need to bear in mind that this data entry should be well prepared and processed in a proper way because the goal is to use many other different data format files. Then by merging these data, the final required format can be reached as a photo image 3D DEM surface of larger terrains. Preparation of data sources SRTM DEM and Google Earth print screen photo image as data entry before starting the automatizing processes in FME. 


\subsubsection{Preparation of DEM file format as USGS (United States Geological Survey) DEM file data from data sources of SRTM DEM file format.}

Firstly, we get the source data file format as SRTM file for area of Europe SE (area of (5 Arc degree) $\mathrm{x}$ (5 Arc degree)) downloaded from its source origin [3] and then we read this data through GM.

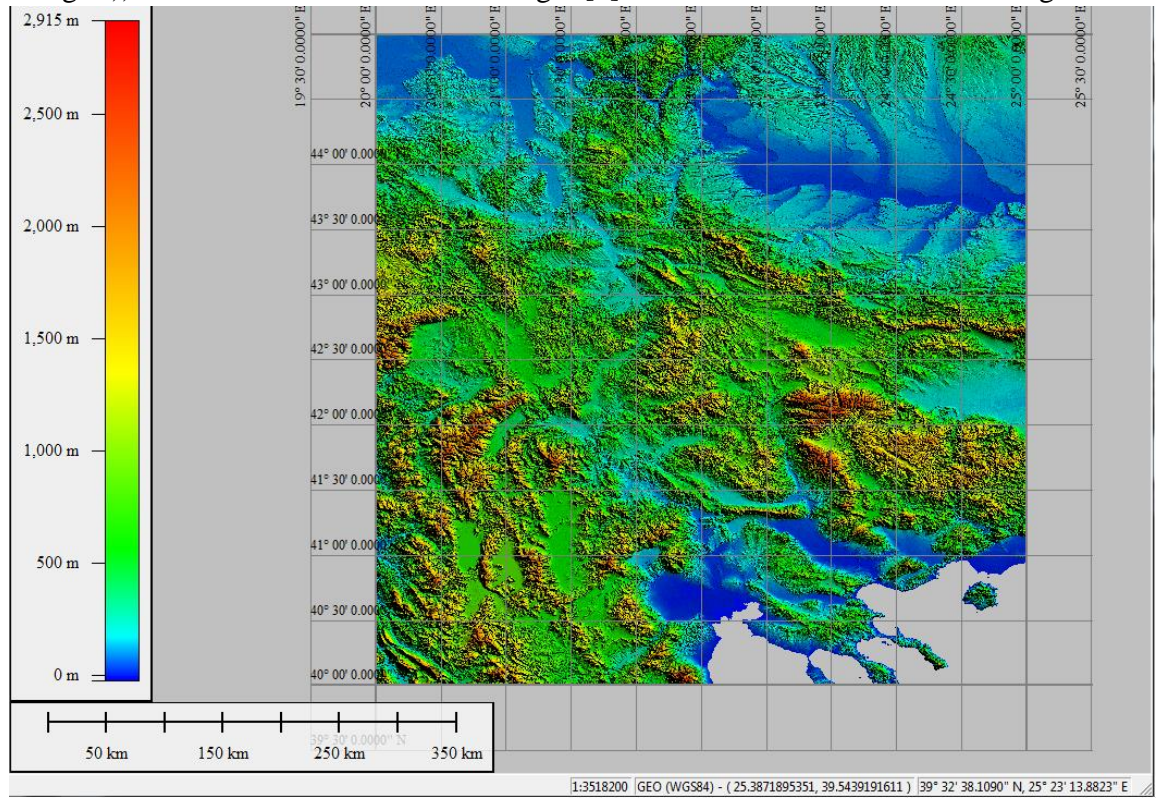

Fig. 12. SRTM (3 arc degree) data for area of Europe (South-East).

From GM we then extract the output format as USGS DEM for the bounding box of the territory of Kosovo. The coordinate system in GM used for this purpose was WGS84.
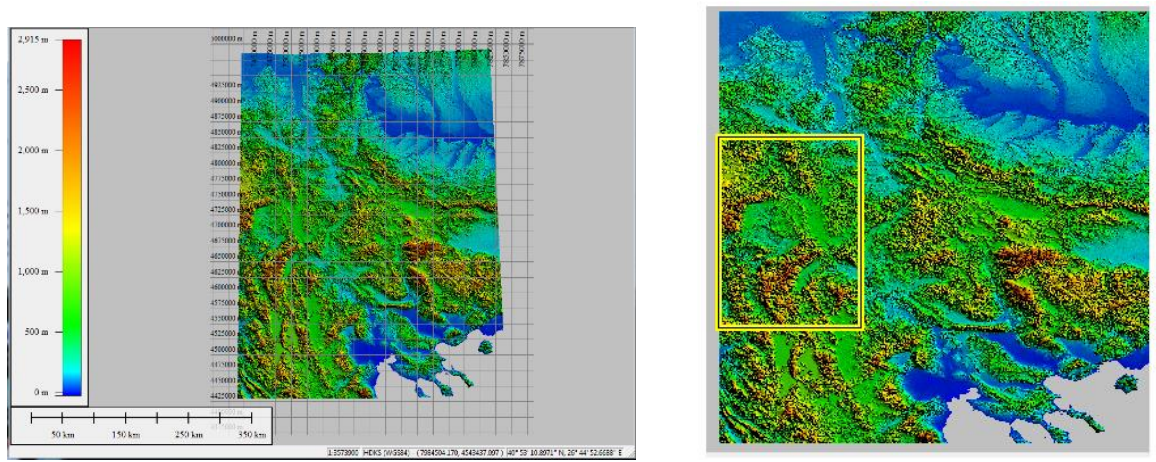

Fig. 13. Extracting by bounding box of desired area(territory of Kosovo)

\subsubsection{Preparation of "Print screen Photo images" of Google Earth for overlapping in USGS DEM format file.}

Photos of print screen images of part of the territory of Kosovo are taken from the screen shoot on the orthogonal Google Earth screen view. First the Google earth was established in an orthogonal projection exactly in a 2D projection and all back elements of views of Google Earth are cleaned and left to show the only quadratic point market places. These quadratic point market places will represent the base points for geo-referencing photo images and to be formed as one photo image. Merging of the pieces of photos can be done with graphic design software or a solution by any software. In our case geo-referencing of the merged photo will be done by GM and will be projected with our chosen coordinate system of GM. 


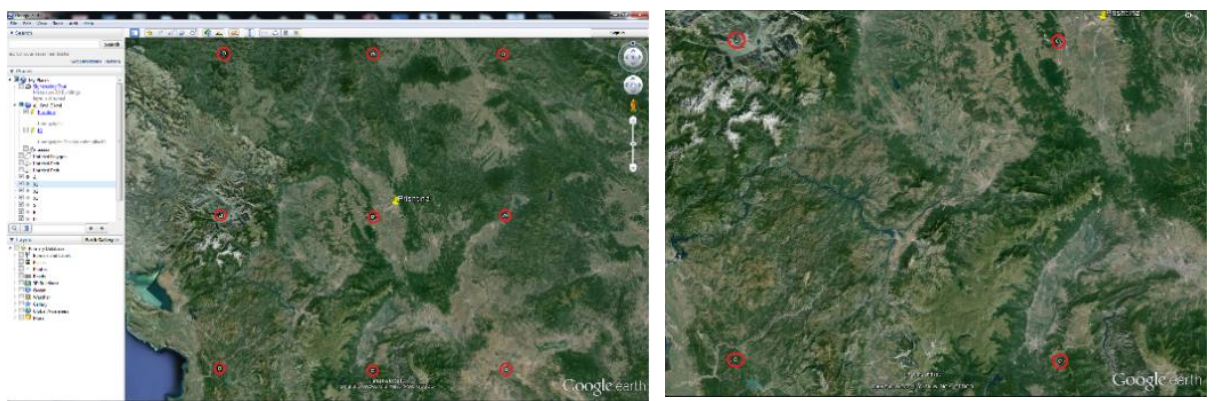

a) Print screen (Very low resolution)

b) Print screen by each quadrants (Much better resolution)

Fig. 14. a) And b) Getting the orthogonal picture print screen of desired area(territory of Kosovo) from Google Earth.

From GM, SRTM file format will be inserted and shape quadratic polygon for correspondent bounding box of pieces of photos aligned to the correspondent coordinate system. Each piece of photos will be inserted due to the process of geo-referencing by correspondent quadratic polygon coordinates of the correspondent photo.

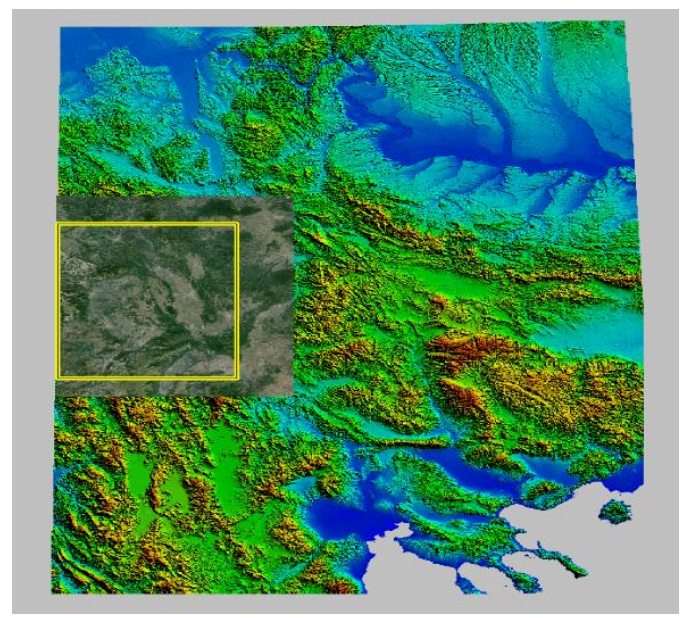

Fig. 15. Overlapping the geo-referenced picture.

The output of export for the elevation data from SRTM data will be done using the USGS DEM format for area (approximately the area of $3 \times 3$ degree) of the chosen bounding box for the territory of Kosovo, while for the output format for raster for the same bounding box. jpg format will be used. In the FME, the input readers for USGS, jpg and MapInfo format files will be inserted. From USGS data, a logical solution to create the TIN surface from existing functional tools for these purpose, need to be created, and also clippers from clipping of raster image and then be dropped to the created surface from USGS 3D model. The MapInfo vector format will be converted to the process of rasterization and then joined to the new created raster which will be the final raster for dropping to the $3 \mathrm{D}$ surface. The output chosen reader can be $3 \mathrm{ds}$, obj or 3Dpdf file format of 3D surface data model.

The input data: Derived USGS DEM elevation; ".jpg" image raster file and MapInfo vector file 


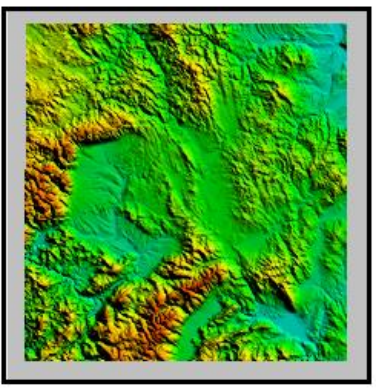

a) Derived USGS DEM file

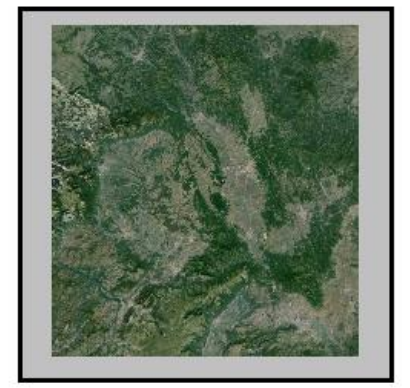

b) Image raster file

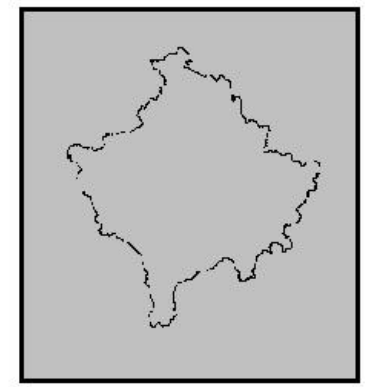

c) MapInfo vector file

Fig. 16. a), b) and c) Input data for workflow in FME

The work flow process:

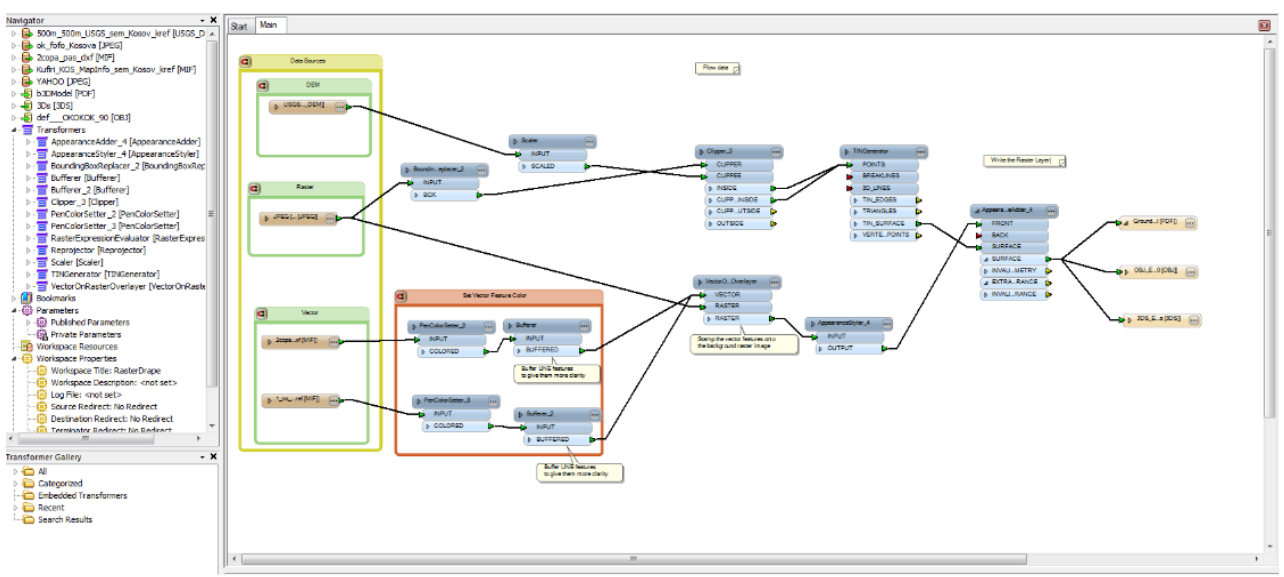

Fig. 17. The workflow: Input data, transformers and output data in FME

The output product: 3D DEM surface of Kosovo.

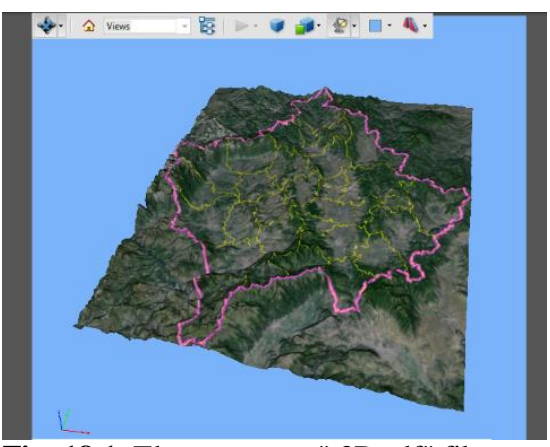

Fig. 18.1. The output as ".3Dpdf" file

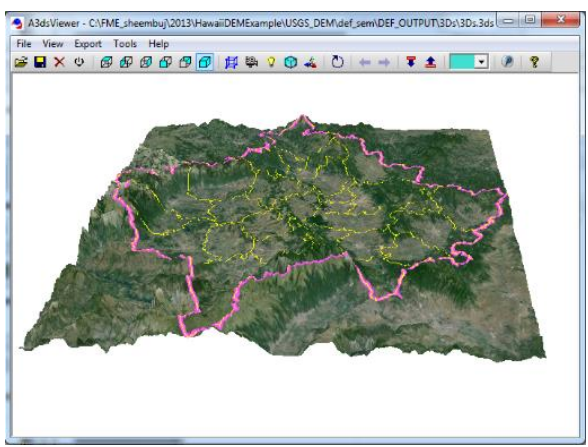

Fig. 18.2. The output as ".3ds" file 


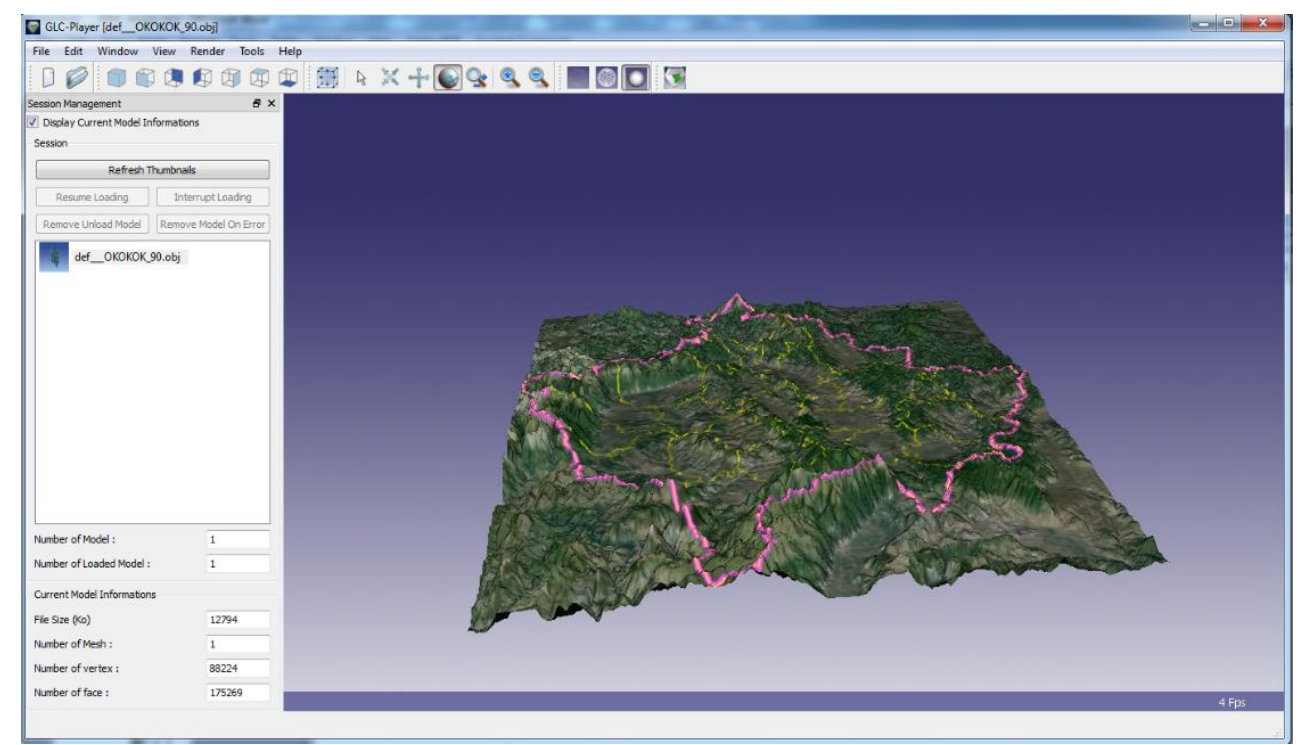

Fig. 18.3. The output as ".obj" file

The result in the editor of FME software:

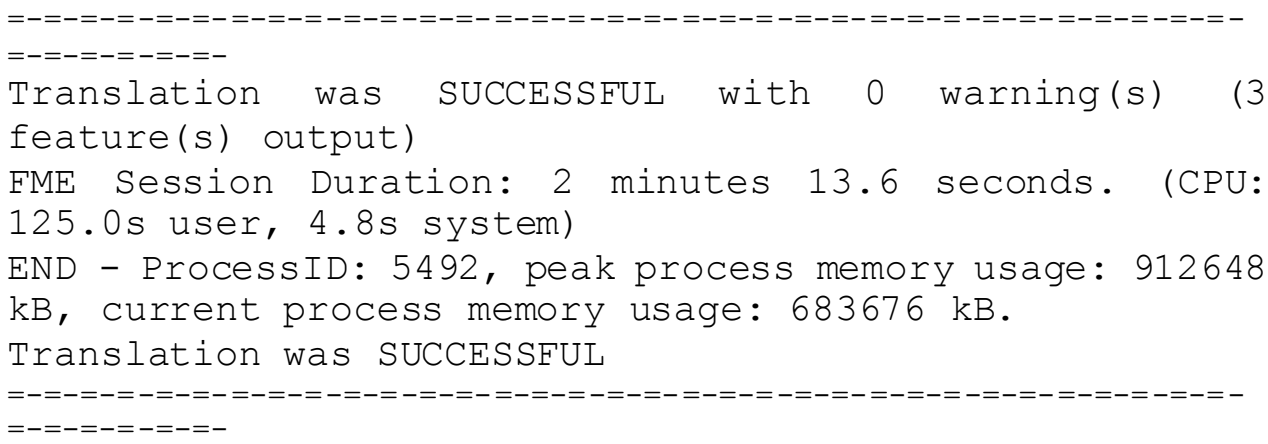

\section{Conclusion}

FME provides unlimited flexibility in data model transformation and distribution and also format support for data translation and integration. By using the Safe FME, the FME can converts data between over 300 formats of data such: GIS, CAD (AutoCAD), raster, Point cloud, 3D, BIM, XML, cloud, non-spatial, and database formats and it has over 400 transformers for manipulating data content and structure. Therefore with the transformers integrated within FME we can create 3D models out of 2D data, that will give the most accurate picture of what is happening, then share this informative 3D model with stakeholders in their 3D format of choice such as 3D PDF, 3ds etc.

Hence FME is a complete spatial ETL(Extract, Transform and Load) solution that enables the processing of bigger data known as GIS data and which enables to quickly translate, transform, integrate and distribute spatial data.

\section{References}

1. Safe FME: http://www.safe.com/(2013)

2. GPS: http://www.garmin.com/ 
International Conference on Civil Engineering, Infrastructure and Environment, Nov 2015

3. SRTM (Shuttle Radar Topography Mission) 3 arc second DEM:

http://www.viewfinderpanoramas.org/(2013); http://ws.csiss.gmu.edu/DEMExplorer/(2014)

4. Global Mapper: www.globalmapper.com(2012);

http://www.bluemarblegeo.com/ (2014)

5. DEM: http://vterrain.org/Elevation/(2014)

http://en.wikipedia.org/wiki/Digital_elevation_model(2014)

6. Google Earth: https://earth.google.com/(2012)

7. Telia, S.: "Producing Digital Elevation Models Using Global Mapper".

http://pbisotopes.ess.sunysb.edu/ (2014) 